\title{
Presentation on understanding uroflowmetry
}

\section{Perspective}

\section{Santron uroflowmeters}

\section{What is uroflowmetry?}

i. The Urinary Flow reflects the final result of the micturiction process consisting of Detrusor Function, bladder neck opening and the Urethral Conductivity.

ii. Uroflowmetry is a simple, non-invasive and initial investigating test that measures the flow rate and Volume of the Urine Stream.

iii. A graphical plot of Flow rate v/s Time along with certain parameters calculated from the test data is used to diagnose urinary outflow obstruction or lower urinary tract dysfunction.

iv. Santron Uroflowmeter is the most reliable, economical and accurate device available to perform the Uroflowmetry test.

v. Uroflowmetry may be combined with simultaneous Santron Uroflowmeter recording of Bladder, Abdominal \& Detrusor pressures, Sphincter EMG for complete bladder function evaluation. This test is Called Urodynamics (Figure 1).

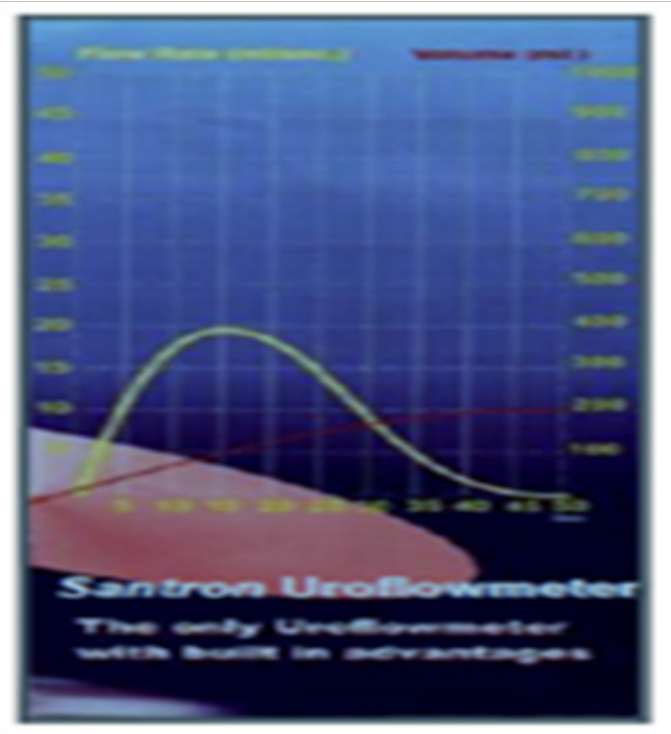

Figure I What is Uroflowmetry?

\section{Why is uroflowmetry performed?}

Uroflowmetry is a quick, non-invasive test that provides valuable feedback about the health of the lower urinary tract. It is commonly performed to determine if there is obstruction to normal urine outflow. Some of the Medical conditions that can alter the normal flow of urine are:

I. Benign prostatic hyperplasia (BPH) - a benign enlargement of the prostate gland that usually occurs in men over age 50 . Enlargement of the prostate interferes with normal passage of urine from the bladder. If left untreated, the enlarged prostate can obstruct the bladder completely.
Volume 3 Issue 6 - 2016

\author{
Narendra Sancheti \\ Medical Devices, India
}

Correspondence: Narendra Sancheti, Medical Devices, Santron Meditronic, Pune, India, Tel 9422035434, Email narendra.sancheti@gmail.com

Received: October 28, 2016| Published: December 8, 2016

II. Cancer of the prostate, or bladder tumor.

III. Urinary incontinence - involuntary release of urine from the bladder.

IV. Urinary blockage - obstruction of the urinary tract can occur for many reasons along any part of the urinary tract from kidneys to urethra. Urinary obstruction can lead to a backflow of urine causing infection, scarring, or kidney failure if untreated.

V. Neurogenic bladder dysfunction.

VI. Frequent urinary tract infections (UTI's).

\section{How is uroflowmetry performed?}

Uroflowmetry is performed by asking the patient to urinate into the funnel that is placed on top of a collection jar. Santron Uroflowmeter sensor then Measures the flow rate $(\mathrm{ml} / \mathrm{sec}$.) of the Urine Stream online along with the voided volume of urine collected in the Jar. The time taken to void (pass urine) is also recorded. This information is then converted into a graph along with certain calculated Uroflow parameters. The shape of Flow pattern (graph), along with the calculated Uroflow parameters helps the physician to interpret \& evaluate the function of the lower urinary tract and then determine if there is an obstruction of normal urine outflow.

\section{Uroflowmetry parameters}

i. Voided volume (ml): The total volume collected in the measuring jar during the voiding (recording) period.

ii. Average flow rate $(\mathrm{ml} / \mathrm{sec})$ : It is the average of the total flow during the voiding time.

iii. Max Flow rate $(\mathrm{ml} / \mathrm{sec})$ : Maximum value of free flow rate, during the voiding period. Max Flow rate is also called as Peak Flow rate.

iv. Computed Max Flow rate ( $\mathrm{ml} / \mathrm{sec})$ : It is the computed value of the sustained Max Flow rate as compared to the measured Max. Flow rate.

v. Time to Max Flow rate (seconds): It is the time at which Max Flow rate occurs.

vi. Voiding time (seconds): Time taken by the person to void. It also includes post micturition drops only if the flow rate is greater than $2 \mathrm{ml} / \mathrm{sec}$. It is measured in seconds. 
vii. Flow time (seconds): The time taken by the patient between the start of voiding and the first time the voiding flow rate reaches zero is called the flow time. It is measured in seconds

viii. Delay time (seconds): It is the time from request to urinate until the actual micturition, or from the existence of a considerable urge to urinate until the beginning of voiding.

ix.Interval Time (second): It is the total time during the entire voiding time, The Patient have paused voiding.

x. Computed Average Flow Rate: It is the average flow rate, recalculated taking into consideration the interval Time.

\section{Interpretation of Uroflowmeter report}

Normal Flow curve decreased Maximum flow and prolonged voiding time typically seen in $\mathrm{BPH}$ conditions (Figure 2).
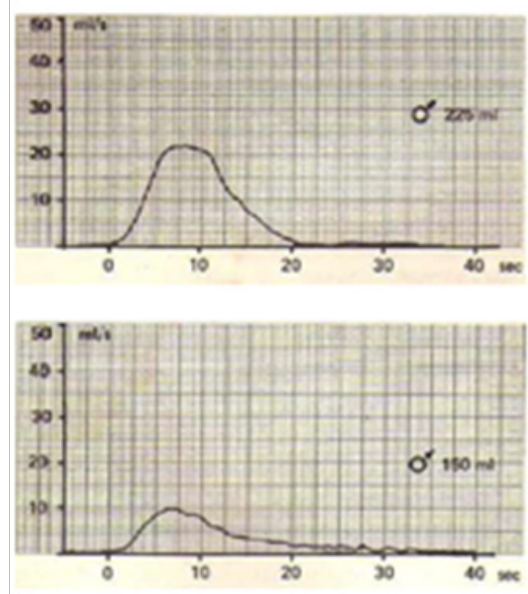

Figure 2 Interpretation of Uroflowmeter report.

I. Interpretation of Uroflowmeter report: Prolonged time to maximum flow and decreased maximum flow rate as seen in bladder neck rigidity. Box like flow curve pattern with low flow rate typically due to Urethral Stricture (Figure 3).
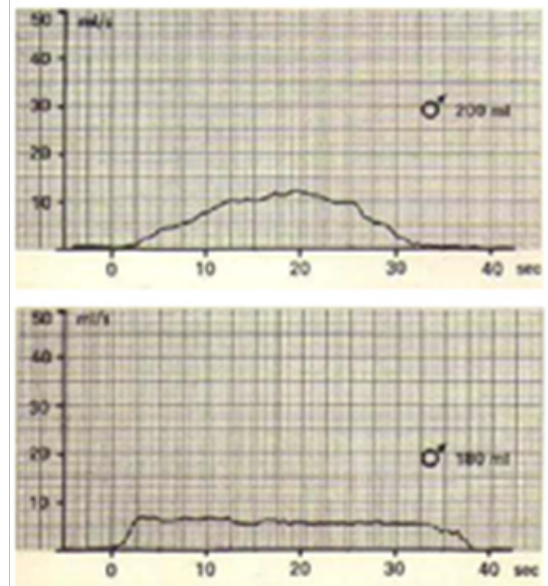

Figure 3 Interpretation of Uroflowmeter report.

II. Interpretation of Uroflowmeter report: Irregular flow curve due to Detrusor contraction combined with abdominal straining or fluctuating obstruction in the lower track. Abrupt decrease of flow rate due to a brief contraction of the external urethral sphincter. This is most likely to be caused by patient discomfort and does not imply the existence of Detrusor sphincter dyssynergia (Figure $4)$.
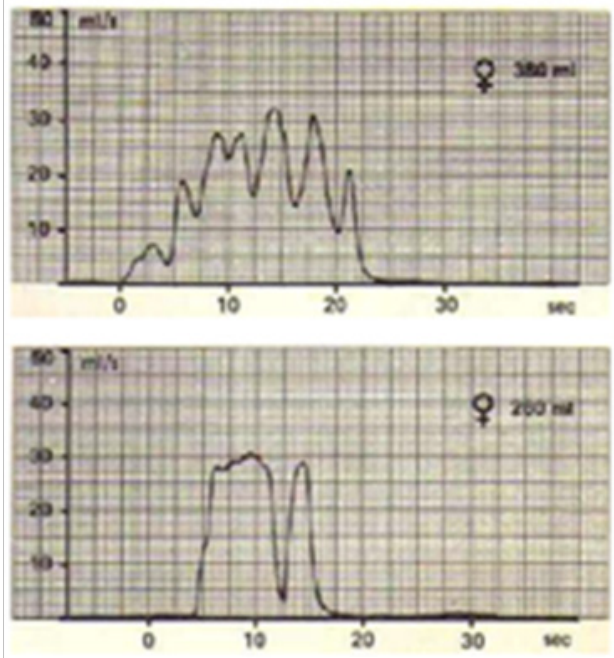

Figure 4 Interpretation of Uroflowmeter report.

III. Interpretation of Uroflowmeter report: Intermittent flow curves as seen in abdominal straining without Detrusor contraction. Extremely high maximum flow rate and brief voiding time as seen in female bladder base insufficiency (Figure 5).
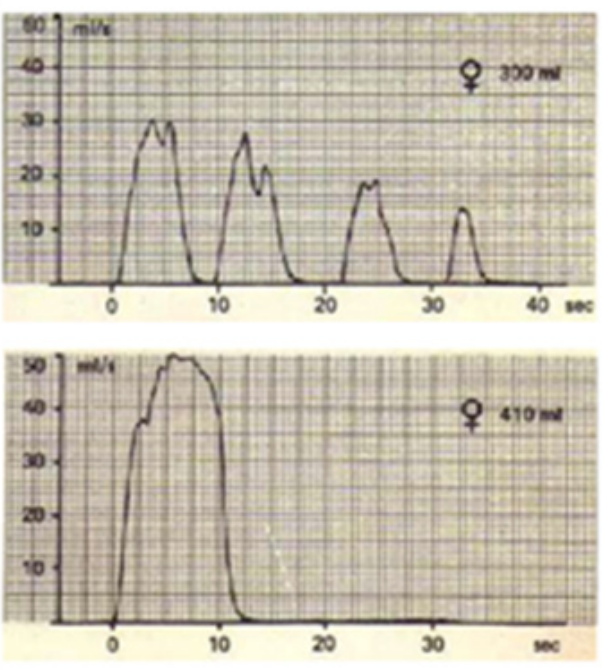

Figure 5 Interpretation of Uroflowmeter report.

\section{Factors affecting a uroflowmetry test}

i. Patient co-operation is essential. Psychological discomfort has to be minimized in order to obtain a proper voiding representation of the habitual pattern during the flow measurement. Santron Uroflowmeters have a unique remote mounted sensor, due to which the patient is able to void in privacy, thus avoiding the psychosomatic side effects, which might influence the measurement results. 
ii. Certain factors or conditions may interfere with the accuracy of Uroflowmetry. These factors include, but are not limited to, the following:

iii. Straining with urination.

iv. Body movement during urination.

v. Certain medications that affect bladder and sphincter muscle tone.

\section{Patient preparation before conducting Uroflowmetry} test

I. A physician should explain the procedure of conducting the Uroflowmetry test to the Patient.

II. Generally, no prior preparation, such as fasting or sedation, is required.

III. The patient is instructed to drink about 3-4 glasses of water a couple of hours before the test is performed to ensure that his bladder is full. In addition, he should not empty his bladder before arriving for the Uroflowmetry test.

IV. Based on the patient's medical condition, the physician may request other specific preparation.

\section{After the Uroflowmetry test}

Generally, there is no special type of care required following a Uroflowmetry Test. However, the physician may give the patient additional or alternate instructions after the Uroflowmetry test, depending on the situation.

\section{Is Uroflowmetry Test risky?}

Because Uroflowmetry is a noninvasive procedure it is safe and has no side effects for the patient.

Disclaimer Note: The content provided here is for informational purposes only and was not designed to diagnose or treat a health problem or disease, or replace the professional medical advice one receives from his physician. Please consult your physician with any questions or concerns you may have regarding your condition.

\section{Acknowledgments}

None.

\section{Conflicts of interest}

The author declares there is no conflict of interest. 